\title{
RESULTADOS PRELIMINARES EN FERTILIZACIONES DE APOYO PARA Eucalyptus globulus
}

\author{
Manuel Toral Ibáñez (*) \\ Patricio Rojas Vergara (*)
}

\section{RESUMEN}

Se evalúan dos ensayos de fertilización en plantaciones recién establecidas de $\boldsymbol{E}$. globulus. Los ensayos en referencia fueron establecidos en 1986 por INFOR-CORFO en convenio con las Empresas Forestales FORVESA y Forestal Colcura, predios El Almendro y Colcura, respectivamente.

En cada ensayo se utilizó un diseño de bloques al azar que consideró diferentes dosis de los siguientes fertilizantes: Urea, Superfosfato Triple, Sulfato de Potasio, Boronatrocalcita y Acido Bórico.

En El Almendro se obtuvieron respuestas positivas a la fertilización con Nitrógeno y Fósforo. En Colcura las mejores respuestas se obtuvieron sólo con Nitrógeno.

\footnotetext{
ABSTRACT

The application of fertilizers at establishment of Eucalyptus globulus plantations are evaluated. Two trials were established by INFOR in 1986, with the cooperation of two forest companies: FORVESA and Forestal Colcura.

$A$ randomized complete block design was used in each case to test different dosis and combinations of the following fertilizer: Urea, Triple Superphosphate, Potasium Sulfate, Boronatrocalcite and Boric Acid.

In one site the best response was produced by the application of $N$ and $P$, where in the other one the sole application of $N$ produced the best results.
}

$\left(^{*}\right) \quad$ Ingenieros Forestales, División Silvicultura, Instituto Forestal, Huérfanos 554, Piso $4^{\circ}$, Santiago - Chile. 


\section{INTRODUCCION}

Las plantaciones de Eucalyptus globulus en Chile, cubren una superficie cercana a las 64.000 ha, concentradas principalmente en la V y VIII Regiones del país.

Aunque $\boldsymbol{E}$. globulus ha sido plantado desde comienzos de siglo, sólo en los últimos años se han realizado prácticas silviculturales que promueven un mejor desarrollo de esta especie. Dentro de estas prácticas silvícolas se encuentra la fertilización de plantaciones. Es así como se han instalado algunos ensayos experimentales de fertilización, y a veces éstos se han combinado con preparación de suelo y control de malezas (PRADO y WRANN, 1988).

Los resultados preliminares en fertilizaciones de apoyo logrados en otros países han sido positivos, lo que ha permitido que gran parte de las empresas del sector estén incorporando esta práctica silvicultural al establecer la plantación (SCHÖNAU y HERBERT, 1988).

El presente trabajo evalúa la supervivencia y. desarrollo de $\boldsymbol{E}$. globulus considerando diferentes dosis y tipo de fertilizantes, utilizados en fertilizaciones de apoyo. La experiencia en referencia se llevó a cabo por INFOR-CORFO en Convenio con Forestal Río Vergara y Forestal Colcura en los predios El Almendro y Colcura, respectivamente.

\section{MATERIAL Y METODO}

\section{Material}

\section{Ubicación y Caracterización de los Ensayos}

El Almendro está ubicado en la IX Región, provincia de Malleco, comuna de Renaico, con coordenadas $37^{\circ} 30^{\prime} 45^{\prime \prime}$ Lat. Sur y $72^{\circ} 45^{\prime}$ Long. Oeste. Corresponde a la Unidad Edofoclimática Concepción. (Univ. de Chile - INFOR, 1974).

Colcura está ubicado en la VIII Región, provincia de Concepción, comuna de Lota, con coordenadas $37^{\circ} 05^{\prime}$ Lat. Sur y $73^{\circ} 10^{\prime}$ Long. Oeste. Corresponde a la Unidad Edafoclimática Lebu.

Las características de los ensayos El Almendro y Colcura, se detallan a continuación.

\section{Ensayo El Almendro} mientos.

Se utilizó un diseño en bloques al azar con cuatro repeticiones y con los siguientes trata-
1. Testigo
2. $\mathrm{N}_{1}$
3. $\mathrm{N}_{2}$
4. $\mathrm{N}_{1} \mathrm{P}_{1}$
5. $\mathrm{N}_{1} \mathrm{P}_{1} \mathrm{~B}_{1} \mathrm{~K}_{1}$
6. $\mathrm{N}_{1} \mathrm{~B}_{1}$
7. $\mathrm{N}_{1} \mathrm{~K}_{1}$
8. $\mathrm{N}_{2} \mathrm{P}_{1}$
9. $\mathrm{N}_{2} \mathrm{P}_{1} \mathrm{~B}_{1} \mathrm{~K}_{1}$
10. $\mathrm{N}_{2} \mathrm{~B}_{1}$
11. $\mathrm{N}_{2} \mathrm{~K}_{1}$
12. $\mathrm{B}_{1} \mathrm{P}_{1} \mathrm{~K}_{1}$
13. $\mathrm{B}_{2} \mathrm{~K}_{1}$
14. $B_{1} P_{1}$

Las dosis por planta para cada tratamiento fueron las siguientes:
$\mathrm{N}_{1}=50 \mathrm{~g}$ de Urea
$\mathrm{N}_{2}=80 \mathrm{~g}$ de Urea
$P_{1}=40 \mathrm{~g}$ de Superfosfato Triple
$\mathrm{B}_{1}=30 \mathrm{~g}$ de Boronatrocalcita
$\mathrm{K}_{1}=50 \mathrm{~g}$ de Sulfato de Potasio 


\section{Ensayo Colcura}

Se utilizó un diseño de bloques al azar con seis repeticiones y con los siguientes tratamientos.
1. Testigo
8. $\mathrm{N}_{2} \mathrm{~B}_{1}$
2. $\mathrm{B}_{1}$
3. $\mathrm{B}_{2}$
9. $\mathrm{N}_{2} \mathrm{~B}_{2}$
4. $\mathrm{N}_{1}$
10. $\mathrm{P}_{1}$
5. $\mathrm{N}_{1} \mathrm{~B}_{1}$
11. $P_{1} B_{1}$
6. $\mathrm{N}_{1} \mathrm{~B}_{2}$
12. $P_{1} B_{2}$
7. $\mathrm{N}_{2}$
13. $\mathrm{N}_{1} \mathrm{P}_{1}$
14. $\mathrm{N}_{1} \mathrm{P}_{1} \mathrm{~B}_{1}$

Las dosis por planta para cada tratamiento fueron las siguientes:
$\mathrm{N}_{1}=50 \mathrm{~g}$ de Urea
$\mathrm{N}_{2}=100 \mathrm{~g}$ de Urea
$\mathrm{P}_{1} \quad=40 \mathrm{~g}$ de Superfosfato Triple
$\mathrm{B}_{1}=20 \mathrm{~g}$ de Acido Bórico
$\mathrm{B}_{2}=40 \mathrm{~g}$ de Acido Bórico

Tanto en Colcura como en El Almendro los fertilizantes fueron aplicados en forma localizada a $20 \mathrm{~cm}$ de profundidad y en 2 zanjas ubicadas paralelamente a $25 \mathrm{~cm}$ de la planta.

Al final de la temporada de plantación se aplicó el $50 \%$ de la dosis especificada para cada tratamiento (Septiembre 1986). El otro 50\% se aplicó en el mes de Septiembre de 1987. En Mayo de 1988 se realizó un tercer control que dio origen a este trabajo.

\section{Metodologia de Análisis}

Los ensayos se analizaron considerando tres metodologías, a saber:

1. Análisis estad ístico del diseño experimental.

2. Cálculo del Optimo Provisional Experimental.

3. Selección de variables. Método de regresión paso a paso. Stepwise.

El método 1 "Análisis estadístico del diseño experimental", consiste en determinar si las medias de los incrementos de la variable de estado en estudio de cada tratamiento son iguales o difieren entre sí. Para ello se emplea el análisis de varianza (ANDEVA) con un nivel de significación igual a 0.05 . En caso de existir diferencias significativas se realizó una comparación entre todos los pares de tratamientos o Test de comparaciones múltiples de Tukey.

La homogeneidad de las varianzas se determinó empleando la prueba de Bartlett. Cuando éstas resultaron heterogéneas se procedió a una transformación logarítmica.

Los porcentajes de supervicencia fueron transformados a la función $\mathrm{y}=$ arcoseno

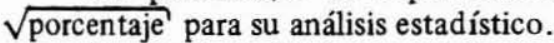

El método 2, "Cálculo del Optimo Provisional Experimental (OPE)", descrito por GONZALEZ et al (1973) consiste en determinar niveles óptimos foliares entre tres nutrientes. Para tales efectos los rangos de dispersión del porcentaje foliar de cada nutriente se subdivide en 5 clases asignándole a cada uno su rendimiento relativo. Para el cálculo del rendimiento relativo se consideró el índice $D^{2} \mathrm{H}$, tomando como base 100 el tratamiento con mayor rendimiento absoluto. Se define el nivel óptimo foliar del nutriente en el promedio de la clase que presenta mayor rendimiento relativo. 
El método 3, "Método de regresión paso a paso", consiste en seleccionar a través de regresiones sucesivas las variables independientes por orden de importancia que son significativas y explicativas del comportamiento de la variable dependiente.

Como variables dependientes se definieron las siguientes:

$\mathrm{DC}^{2} \mathrm{H}$ : Indice de crecimiento en volumen. Diámetro del cuello de la planta al cuadrado multiplicado por su altura y dividido por $100\left(\mathrm{~cm}^{3}\right)$.

DC : Diámetro de Cuello $(\mathrm{cm})$.

$\mathrm{H} \quad$ : Altura (m).

Como variables independientes se de finieron las siguientes:

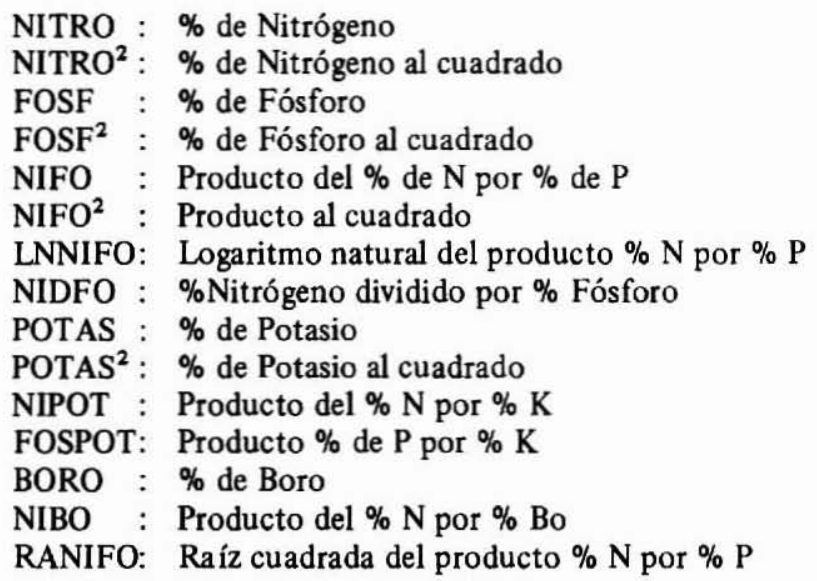

Como las variables independientes alcanzan a un alto número se optó por utilizar del paquete estadístico "Statgraphics", el método de regresión paso a paso denominado "Forward" o procedimiento paso a paso estándar (Método $\mathrm{F}$ ). A través de este método las variables entran o son removidas de la ecuación según su valor de $\mathrm{F}$. El valor de $\mathrm{F}$ se fijó en 4,67 , debido a que sólo se contó con 14 valores para el cálculo de cada regresión, aunque por defecto el paquete "Statgraphics" fija un valor de $F=4,0$.

\section{RESULTADOS}

Para una mejor comprensión de los resultados, éstos se presentan de acuerdo a las diferentes metodologías planteadas.

\section{Método 1. Análisis Estadistico del Diseño Experimental}

\section{El Almendro}

Los resultados del ensayo se presentan en la Tabla 1 que entrega la supervivencia, los incrementos medios de las variables Diámetro de Cuello, DC (cm); Altura, H (m) y Volumen expresado a través del índice $\mathrm{DC}^{2} \mathrm{H}\left(\mathrm{cm}^{3}\right)$. 


\section{TABLA 1 \\ SUPERVIVENCIA E INCREMENTOS MEDIOS \\ DE DC, H Y DC ${ }^{2}$ H. 1986 - 1988 \\ (Ensayo: Predio El Almendro - FORVESA)}

\begin{tabular}{|c|c|c|c|c|}
\hline TRATAMIENTOS & $\begin{array}{l}\text { SUPERVIV. \% } \\
\text { (1988) }\end{array}$ & $\begin{array}{l}\mathrm{DC} \\
(\mathrm{cm})\end{array}$ & $\underset{(\mathrm{m})}{\mathbf{H}}$ & $\begin{array}{l}\mathrm{DC}^{2} \mathrm{H} \\
\left(\mathrm{cm}^{3}\right)\end{array}$ \\
\hline 1. Testigo & 63,9 & $2,9 \mathrm{~b}$ & 1,4 & $25,3 \mathrm{~b}$ \\
\hline 2. $\mathrm{N}_{1}$ & 69,5 & $2,5 \mathrm{~b}$ & 1,3 & $16,3 \mathrm{bc}$ \\
\hline 3. $\mathrm{N}_{2}$ & 58,3 & $3,4 \mathrm{~b}$ & 1,2 & $22,6 \quad b$ \\
\hline 4. $\mathrm{N}_{1} \mathrm{P}_{1}$ & 63,9 & $3,1 \mathrm{~b}$ & 1,4 & $25,7 \mathrm{~b}$ \\
\hline 5. $\mathrm{N}_{1} \mathrm{P}_{1} \mathrm{~B}_{1} \mathrm{~K}_{1}$ & 86,1 & 4,4 a & 2,1 & 66,9 a \\
\hline 6. $\mathrm{N}_{1} \mathrm{~B}_{1}$ & 69,5 & $2,8 \mathrm{~b}$ & 1,5 & $26,2 \mathrm{~b}$ \\
\hline 7. $\mathrm{N}_{1} \mathrm{~K}_{1}$ & 86,1 & $3,2 \mathrm{~b}$ & 1,6 & $30,9 \mathrm{~b}$ \\
\hline 8. $\mathrm{N}_{2} \mathrm{P}_{1}$ & 77,8 & $3,7 \mathrm{c}$ & 1,8 & $42,2 \mathrm{~b}$ \\
\hline 9. $\mathrm{N}_{2} \mathrm{P}_{1} \mathrm{~B}_{1} \mathrm{~K}_{1}$ & 80,6 & $2,8 \mathrm{~b}$ & 1,9 & $47,3 \mathrm{c}$ \\
\hline 10. $\mathrm{N}_{2} \mathrm{~B}_{1}$ & 75,0 & $3,6 \mathrm{~b}$ & 1,9 & $35,8 \mathrm{~b}$ \\
\hline 11. $\mathrm{N}_{2} \mathrm{~K}_{1}$ & 72,3 & $2,9 \mathrm{~b}$ & 1,5 & $29,1 \mathrm{~b}$ \\
\hline 12. $\mathrm{B}_{1} \mathrm{P}_{1} \mathrm{~K}_{1}$ & 77,8 & $2,3 \mathrm{~b}$ & 1,2 & $40,2 \mathrm{~b}$ \\
\hline 13. $\mathrm{B}_{2} \mathrm{~K}_{1}$ & 72,3 & $2,3 \mathrm{bc}$ & 1,2 & $17,4 \mathrm{~b}$ \\
\hline 14. $\mathrm{B}_{1} \mathrm{P}_{1}$ & 83,4 & $2,2 \mathrm{bc}$ & 2,0 & $12,8 \mathrm{bc}$ \\
\hline $\begin{array}{l}\text { Nivel de significación } \\
\text { al } 0,05\end{array}$ & $\begin{aligned} \mathrm{Fc} & =0,097 \mathrm{NS} \\
\mathrm{Ft} & =2,016\end{aligned}$ & $\begin{array}{l}\mathrm{Fc}=2.620 \mathrm{~S} \\
\mathrm{Ft}=2,016\end{array}$ & $\begin{array}{l}\mathrm{Fc}=1.529 \mathrm{NS} \\
\mathrm{Ft}=2,016\end{array}$ & $\begin{array}{l}\mathrm{Fc}=2.767 \mathrm{~S} \\
\mathrm{Ft}=2,016\end{array}$ \\
\hline
\end{tabular}

Letras distintas significa que hay diferencias significativas al 0,05 .

Del análisis de la Tabla 1, se desprende que no existen diferencias estad ísticamente significativas para la supervivencia y altura entre los tratamientos. Existen para las variables diámetro de cuello e índice de crecimiento $\mathrm{DC}^{2} \mathrm{H}$.

Los mayores incrementos en volumen se presentan en los tratamientos 5,8 y 9 . En dos de ellos se han aplicado los 4 elementos, N P K B, que en el caso del tratamiento 5 producen una respuesta significativamente superior. Al analizar el conjunto de los tratamientos se concluye que la combinación NP sería la que produce la mayor respuesta, que en el caso de los tratamientos 5 y 9 se ve incrementada por la presencia de los otros dos elementos.

\section{Colcura}

Los resultados de este ensayo se presentan en la Tabla 2, que entrega los incrementos medios de las variables Diámetro de Cuello, DC $(\mathrm{cm})$; Altura, H $(\mathrm{m})$ y Volumen expresado por el indice $\mathrm{DC}^{2} \mathrm{H}$. 
TABLA 2

\section{SUPERVIVENCIA E INCREMENTOS MEDIOS \\ DE DC, H Y DC ${ }^{2}$ H. 1986 - 1988}

(Ensayo: Predio Colcura - Forestal Colcura)

\begin{tabular}{|c|c|c|c|c|}
\hline TRATAMIENTOS & $\begin{array}{c}\text { SUPERVIV. \% } \\
\text { (1988) }\end{array}$ & $\begin{array}{l}\mathrm{DC} \\
(\mathrm{cm})\end{array}$ & $\begin{array}{l}\mathrm{H} \\
(\mathrm{m})\end{array}$ & $\begin{array}{l}\mathrm{DC}^{2} \mathrm{H} \\
\left(\mathrm{cm}^{3}\right)\end{array}$ \\
\hline 1. Testigo & 77,8 & $2,9 \mathrm{c}$ & $2,4 \mathrm{~cd}$ & 65,2 \\
\hline 2. $B_{1}$ & 87,1 & $4,2 \mathrm{bc}$ & $3,2 \mathrm{bcd}$ & 114,9 \\
\hline 3. $\mathrm{B}_{2}$ & 88,9 & $3,3 \mathrm{bc}$ & $2,4 \mathrm{~cd}$ & 70,9 \\
\hline 4. $\mathrm{N}_{1}$ & 77,8 & 5,2 a & 4,1 a & 158,4 \\
\hline 5. $\mathrm{N}_{1} \mathrm{~B}_{1}$ & 85,2 & $4,8 \mathrm{~b}$ & $3,6 \mathrm{~d}$ & 127,3 \\
\hline 6. $\mathrm{N}_{1} \mathrm{~B}_{2}$ & 79,7 & $3,3 \mathrm{bc}$ & $2,5 \mathrm{c}$ & 64,5 \\
\hline 7. $\mathrm{N}_{2}$ & 83,4 & $3,3 \mathrm{bc}$ & $2,8 \mathrm{bcd}$ & 69,3 \\
\hline 8. $\mathrm{N}_{2} \mathrm{~B}_{1}$ & 79,7 & $4,4 \mathrm{bc}$ & $3,3 \mathrm{bcd}$ & 125,1 \\
\hline 9. $\mathrm{N}_{2} \mathrm{~B}_{2}$ & 87,1 & $4,7 \mathrm{bc}$ & $3,7 \mathrm{bcd}$ & 146,8 \\
\hline 10. $P_{1}$ & 68,5 & $3,9 \mathrm{bc}$ & 2,9 bcd & 98,0 \\
\hline 11. $P_{1} B_{1}$ & 72,2 & $4,2 \mathrm{bc}$ & 3,2 bcd & 130,5 \\
\hline 12. $P_{1} B_{2}$ & 62,9 & $4,5 \mathrm{bc}$ & 3,4 bcd & 148,5 \\
\hline 13. $\mathrm{N}_{1} \mathrm{P}_{1}$ & 72,2 & $3,2 \mathrm{bc}$ & $2,7 \mathrm{bcd}$ & 55,9 \\
\hline 14. $N_{1} P_{1} B_{1}$ & 81,5 & $4,0 \mathrm{bc}$ & 3,0 bcd & 85,4 \\
\hline $\begin{array}{l}\text { Nivel de significación } \\
\text { al } 0,05\end{array}$ & $\begin{array}{l}\mathrm{Fc}=1,32 \mathrm{NS} \\
\mathrm{Ft}=1,870\end{array}$ & $\begin{array}{l}\mathrm{Fc}=2,123 \mathrm{~S} \\
\mathrm{Ft}=1,870\end{array}$ & $\begin{array}{l}\mathrm{Fc}=2,343 \mathrm{~S} \\
\mathrm{Ft}=1,870\end{array}$ & $\begin{array}{l}\mathrm{Fc}=1,338 \mathrm{NS} \\
\mathrm{Ft}=1,870\end{array}$ \\
\hline
\end{tabular}

Al comparar los tratamientos se puede apreciar que existen importantes diferencias en la supervivencia y especialmente en el indice de crecimiento $D^{2} \mathrm{H}$, aún cuando las pruebas estadísticas indican que éstas no son significativas.

En este caso las mejores respuestas corresponden a la sola aplicación de $\mathrm{N}$ (Trat. 4) y a su combinación con B (Trats. 8 y 9). La aplicación de P y B también produce un buen crecimiento (Trats. 11 y 12) en cambio la aplicación NP, que en el ensayo anterior entregaba los mejores resultados, produce en este caso una respuesta inferior a la del testigo. Es posible que se haya producido un desbalance en la relación N/P, pudiendo ser ésta la causa de esta baja respuesta. 
M. TORAL I. y P. ROJAS V.

Método 2. Cálculo del Optimo Provisional Experimental (OPE)

En la Tabla 3 se presentan los niveles foliares de N, P, K por tratamiento.

TABLA 3

NIVELES FOLIARES DE N, P, K POR TRATAMIENTO

(Ensayo: El Almendro y Colcura. Mayo 1988)

\begin{tabular}{|c|c|c|c|c|c|c|c|}
\hline \multicolumn{4}{|c|}{ EL ALMENDRO } & \multicolumn{4}{|c|}{ COLCURA } \\
\hline \multirow{2}{*}{ Tratamientos } & \multicolumn{3}{|c|}{ Porcentajes de } & \multirow{2}{*}{ Tratamientos } & \multicolumn{3}{|c|}{ Porcentajes de } \\
\hline & $\mathbf{N}$ & $\mathbf{P}$ & $\mathbf{K}$ & & $\mathbf{N}$ & $\mathbf{P}$ & $\mathbf{K}$ \\
\hline 1. Testigo & 1,75 & 0,08 & 0,35 & 1. Testigo & 1,61 & 0,08 & 0,58 \\
\hline 2. $\mathrm{N}_{1}$ & 2,04 & 0,07 & 0,37 & 2. $B_{1}$ & 1,99 & 0,10 & 0,58 \\
\hline 3. $\mathrm{N}_{2}$ & 1,87 & 0,80 & 0,39 & 3. $\mathrm{B}_{2}$ & 1,88 & 0,08 & 0,59 \\
\hline 4. $\mathrm{N}_{1} \mathrm{P}_{1}$ & 1,85 & 0,09 & 0,31 & 4. $\mathrm{N}_{1}$ & 1,88 & 0,10 & 0,54 \\
\hline 5. $\mathrm{N}_{1} \mathrm{P}_{1} \mathrm{~B}_{1} \mathrm{~K}_{1}$ & 1,97 & 0,09 & 0,37 & 5. $\mathrm{N}_{1} \mathrm{~B}_{1}$ & 1,61 & 0,08 & 0,52 \\
\hline 6. $\mathrm{N}_{1} \mathrm{~B}_{1}$ & 1,85 & 0,07 & 0,31 & 6. $\mathrm{N}_{1} \mathrm{~B}_{2}$ & 1,60 & 0,10 & 0,59 \\
\hline 7. $\mathrm{N}_{1} \mathrm{~K}_{1}$ & 1,83 & 0,08 & 0,37 & 7. $\mathrm{N}_{2}$ & 1,71 & 0,06 & 0,48 \\
\hline 8. $\mathrm{N}_{2} \mathrm{P}_{1}$ & 2,11 & 0,09 & 0,39 & 8. $\mathrm{N}_{2} \mathrm{~B}_{1}$ & 1,74 & 0,08 & 0,52 \\
\hline 9. $\mathrm{N}_{2} \mathrm{P}_{1} \mathrm{~B}_{1} \mathrm{~K}_{1}$ & 2,18 & 0,09 & 0,33 & 9. $\mathrm{N}_{2} \mathrm{~B}_{2}$ & 2,17 & 0,07 & 0,56 \\
\hline 10. $\mathrm{N}_{2} \mathrm{~B}_{1}$ & 2,16 & 0,08 & 0,31 & 10. $P_{1}$ & 1,97 & 0,10 & 0,50 \\
\hline 11. $\mathrm{N}_{2} \mathrm{~K}_{1}$ & 1,82 & 0,09 & 0,45 & 11. $\mathrm{P}_{1} \mathrm{~B}_{1}$ & 1,61 & 0,10 & 0,57 \\
\hline 12. $\mathrm{B}_{1} \mathrm{P}_{1} \mathrm{~K}_{1}$ & 1,81 & 0,10 & 0,40 & 12. $P_{1} B_{2}$ & 2,16 & 0,08 & 0,53 \\
\hline 13. $\mathrm{B}_{2} \mathrm{~N}_{1}$ & 1,67 & 0,08 & 0,33 & 13. $\mathrm{N}_{1} \mathrm{P}_{2}$ & 1,96 & 0,09 & 0,51 \\
\hline 14. $B_{1} P_{1}$ & 1,67 & 0,10 & 0,37 & 14. $\mathrm{N}_{1} \mathrm{P}_{1} \mathrm{~K}_{1}$ & 1,82 & 0,08 & 0,45 \\
\hline
\end{tabular}

A partir de estos valores y del rendimiento relativo en volumen se calculó el Optimo Provisional Experimental, según la metodología propuesta por GONZALEZ $\underline{\text { et }}$ al op. cit. entregando los siguientes resultados.

\section{Ensayo El Almendro}

En la Tabla 4 se presentan los rangos de los niveles de Nitrógeno, Fósforo y Potasio foliar versus su rendimiento relativo promedio, dado por el indice de crecimiento $\mathrm{DC}^{2} \mathrm{H}$. 
TABLA 4

NIVELES DE NUTRIENTES Y SU RENDIMIENTO

RELATIVO PROMEDIO EN VOLUMEN (DC $\left.{ }^{2} \mathrm{H}\right)$

(Ensayo: Predio El Almendro - FORVESA)

\begin{tabular}{|c|c|c|c|c|c|}
\hline \multicolumn{2}{|c|}{ NITROGENO } & \multicolumn{2}{c|}{ FOSFORO } & \multicolumn{2}{c|}{ POTASIO } \\
\hline $\begin{array}{c}\text { Rangos } \\
\text { \% Foliar }\end{array}$ & $\begin{array}{c}\text { Rendim. } \\
\text { Promed. \% } \\
\text { DC }^{\mathbf{2}} \mathbf{H}\end{array}$ & $\begin{array}{c}\text { Rangos } \\
\% \text { P Foliar }\end{array}$ & $\begin{array}{c}\text { Rendim. } \\
\text { P:omed. \% } \\
\text { DC }^{\mathbf{2}} \mathbf{H}\end{array}$ & $\begin{array}{c}\text { Rangos } \\
\% \text { K Foliar }\end{array}$ & $\begin{array}{c}\text { Rendim. } \\
\text { Prumed. \% } \\
\text { DC }^{\mathbf{2}} \mathbf{H}\end{array}$ \\
\hline $1,60-1,72$ & 27,67 & $0,070-0,076$ & 32,07 & $0,310-0,338$ & 46,23 \\
$1,75-1.87$ & 42,71 & $0,077-0,083$ & 40,24 & $0,339-0,367$ & 37,86 \\
$1,89-1,97$ & 73,11 & $0,084-0,090$ & 61,89 & $0,368-0,396$ & 48,42 \\
$1,98-2,07$ & - & $0,091-0,097$ & - & $0,397-0,425$ & 60,05 \\
$2,08-2,18$ & 52,90 & $0,098-0,104$ & 39,62 & $0,426-0,454$ & 34,48 \\
\hline
\end{tabular}

A partir de la Tabla 4 se establecieron los niveles foliares óptimos de N, P y K para las condiciones de este ensayo en particular. En efecto, el nivel óptimo de Nitrógeno se encuentra próximo a $1,93 \%$, el de Fósforo próximo a $0,087 \%$ y Potasio próximo a $0,41 \%$. Estos niveles entregan una relación en porcentaje de $\mathrm{N} / \mathrm{P}=22,18 ; \mathrm{N} / \mathrm{K}=4,70$ y $\mathrm{P} / \mathrm{K}=0,21$.

\section{Ensayo Colcura}

En la Tabla 5 se presentan los resultados de concentración foliar del nutriente y su rendimiento relativo.

TABLA 5

NIVELES DE NUTRIENTES Y SU RENDIMIENTO RELATIVO PROMEDIO EN VOLUMEN $\left(\mathrm{DC}^{2} \mathrm{H}\right)$

(Ensayo: Predio Colcura - Forestal Colcura)

\begin{tabular}{|c|c|c|c|c|c|}
\hline \multicolumn{2}{|c|}{ NITROGENO } & \multicolumn{2}{c|}{ FOSFORO } & \multicolumn{2}{c|}{ POTASIO } \\
\hline $\begin{array}{c}\text { Rangos } \\
\text { \% N Foliar }\end{array}$ & $\begin{array}{c}\text { Rendim. } \\
\text { Promed. \% }_{\mathbf{D C}^{2} \mathbf{H}}\end{array}$ & $\begin{array}{c}\text { Rangos } \\
\text { \% P Foliar }\end{array}$ & $\begin{array}{c}\text { Rendim. } \\
\text { Promed. }_{\mathbf{D C}^{2} \mathbf{H}}\end{array}$ & $\begin{array}{c}\text { Rangos } \\
\text { \% K Foliar }\end{array}$ & $\begin{array}{c}\text { Rendim. } \\
\text { Promed. \% }_{\mathbf{D C}^{2} \mathbf{H}}\end{array}$ \\
\hline $1,600-1,714$ & 57,68 & $0,060-0,068$ & 43,77 & $0,450-0,478$ & 53,92 \\
$1,715-1,829$ & 66,45 & $0,069-0,077$ & 92,67 & $0,479-0,507$ & 52,82 \\
$1,830-1,944$ & 72,38 & $0,078-0,086$ & 65,5 & $0,508-0,536$ & 72,10 \\
$1,945-2,059$ & 56,58 & $0,087-0,095$ & 35,28 & $0,537-0,565$ & 96,33 \\
$2,060-2,174$ & 93,22 & $0,096-0,104$ & 71,51 & $0,566-0,594$ & 56,32 \\
\hline
\end{tabular}


A partir de la Tabla 5 se pueden establecer los niveles óptimos de $\mathrm{N}, \mathrm{P}$ y $\mathrm{K}$ para las condiciones de este ensayo en particular. Es así que el nivel óptimo de Nitrógeno queda fijado en $2,11 \%$; Fósforo en $0,073 \%$ y Potasio próximo a $0,55 \%$. Estos niveles entregan una relación en porcentaje de $\mathrm{N} / \mathrm{P}=28,90 ; \mathrm{N} / \mathrm{K}=3,83$ y de $\mathrm{P} / \mathrm{K}=0,13$.

\section{Método 3. Método de Regresión Paso a Paso}

Numerosos trabajos han establecido relaciones funcionales entre el rendimiento y la concentración de nutrientes utilizando regresiones múltiples, seleccionadas a través de regresiones paso a paso (Stepwise). SCHÖNAU (1981); SCHÖNAU Y HERBERT (1982:83); LAMB (1977); BEVIGE y RICHARDS (1971).

En dichos trabajos generalmente se utiliza el incremento en diámetro, altura o volumen como variables dependientes y como independientes la concentración en porcentaje de los distintos niveles de nutrientes y combinaciones lógicas entre ellos.

Aplicada esta metodología a los ensayos de Colcura y El Almendro, dio los siguientes resultados.

\section{TABLA 6}

\section{SELECCION DE VARIABLES SIGNIFICATIVAS Y PREDICTORAS DE LA VARIABLE DE ESTADO CORRESPONDIENTE}

\begin{tabular}{|c|c|c|c|c|c|c|c|c|}
\hline \multirow{2}{*}{ PREDIO } & \multirow{2}{*}{$\begin{array}{l}\text { VARIABLE DE ESTADO } \\
\text { INCREMENTOS }\end{array}$} & \multirow{2}{*}{\multicolumn{3}{|c|}{ FUNCION. PREDICTORAS }} & \multirow{2}{*}{$\mathrm{r}^{2}$} & \multirow{2}{*}{ E.S.E. } & \multicolumn{2}{|c|}{ RANGO DE NIVEL, DE NUTR. } \\
\hline & & & & & & & $\mathbf{~} \mathrm{N}$ & WP \\
\hline COLCURA & $\begin{array}{l}\text { Volumen } \\
\text { Diámetro de Cuello } \\
\text { Altura }\end{array}$ & $\begin{array}{l}\mathrm{DC}^{3} \mathrm{H} \\
\mathrm{DC} \\
\mathrm{H}\end{array}$ & $\begin{array}{l}=33,6365 \mathrm{~N} \\
=2,1477 \mathrm{~N} \\
=1,66402 \mathrm{~N}\end{array}$ & $\begin{array}{l}\text { NITRO'2 } \\
\text { NITRO } \\
\text { NITRO }\end{array}$ & $\begin{array}{l}0,91 \\
0.97 \\
0,97\end{array}$ & $\begin{array}{r}38,12 \\
0,69 \\
0,50\end{array}$ & $\begin{array}{l}1,60-2,17 \\
1,60-2,17 \\
1,60-2,17\end{array}$ & $\begin{array}{l}- \\
-\end{array}$ \\
\hline EL ALMENDRO & $\begin{array}{l}\text { Volumen } \\
\text { Diámetro de Cuello } \\
\text { Altura }\end{array}$ & $\begin{array}{l}\mathrm{DC}^{2} \mathrm{H} \\
\mathrm{DC} \\
\mathrm{H}\end{array}$ & $\begin{array}{l}=1207,57 \mathrm{~N} \\
=1,6025 \mathrm{~N} \\
=9,4851 \mathrm{~N}\end{array}$ & $\begin{array}{l}\text { NIFO }^{2} \\
\text { NITRO } \\
\text { NIFO }\end{array}$ & $\begin{array}{l}0,91 \\
0,97 \\
0,98\end{array}$ & $\begin{array}{r}10,67 \\
0,52 \\
0,22\end{array}$ & $\begin{array}{ll}1,6 & -2,18 \\
1,6 & -2,18 \\
1,6 & -2,18\end{array}$ & $\begin{array}{c}0,07-0,104 \\
- \\
0,07-0,104\end{array}$ \\
\hline
\end{tabular}

E.S.E. $=$ Error estándar de la estimación.

En la Tabla 6 se puede apreciar que para el ensayo de Colcura las únicas variables independientes que explican el incremento de la variable de estado dependiente correspondiente es NITRO $^{2}$ y NITRO (\% de Nitrógeno foliar al cuadrado y \% de Nitrógeno foliar, respectivamente). El resto de las variables analizadas presentan un bajo valor de $F$, menor a 4,67, no significativo. Para El Almendro las variables independientes seleccionadas según variable de estado son NIFO ${ }^{2}$, NITRO y NIFO (producto al cuadrado de \% Nitrógeno foliar y \% de Fósforo, $\%$ de Nitrógeno y producto del \% de Nitrógeno y Fósforo, respectivamente).

$\mathrm{Si}$ analizamos las funciones, se observa que éstas pasan por el origen y en general indican que a mayor porcentaje de concentración foliar de Nitrógeno o Fósforo el incremento de la variable de estado será mayor. Sin embargo, esto no es totalmente válido ya que la ley de los incrementos decrecientes establece que el incremento es máximo sólo hasta un cierto nivel de nutrientes y que si se incorpora una mayor cantidad la tasa de crecimiento comienza a disminuir gradualmente hasta llegar a un mínimo. En consecuencia, las ecuaciones planteadas sólo son válidas para los rangos de niveles de nutrientes analizados e indicados en la Tabla 6. 


\section{DISCUSION}

A pesar de que la concentración de elementos minerales en las hojas es muy variable (depende de la procedencia, especie, suelo, edad, época de muestreo, condiciones climáticas y posición de las hojas), la absorción de nutrientes realizadas por la planta se refleja invariablemente en el análisis foliar (CABAÑAS, 1982).

En nuestro país, no se han conducido investigaciones orientadas a determinar los valores críticos nutricionales para el género Eucalyptus. Los valores obtenidos en estos ensayos representan una primera aproximación a este tema y se deben considerar como valores referenciales de carácter primario y limitados a las condiciones donde se efectuaron.

SCHÖNAU y HERBERT (1988) indican que para $\boldsymbol{E}$. globulus se obtuvieron respuestas satisfactorias a la fertilización en Australia, Portugal, España, Colombia e India. Las respuestas positivas se han debido a la fertilización con Nitrógeno solo, o a la combinación de Nitrógeno y Fósforo. Coincidente con esto fueron los resultados de los ensayos en Colcura y en El Almendro para cada una de las variables de estado analizadas (Métodos 1,2,3).

Los mismos autores señalan que el Nitrógeno y el Fósforo son muy importantes, siendo el primero la unidad básica constituyente de las prote inas y de la clorofila. Es por ende el responsable del crecimiento foliar de la planta, controlando el crecimiento de la madera. El Fósforo juega un rol fundamental en el metabolismo de la planta. FINKL y SIMONSON (1979) establecen que afecta preferentemente la formación de las semillas, la maduración y calidad de las cosechas y por sobre todo al desarrollo de las raíces, particularmente las raicillas laterales. Esta última función del Fósforo es fundamental en los viveros forestales y en el establecimiento de las plantaciones.

Como la función de estos dos elementos se considera primaria, se han establecido ciertas relaciones entre ellos. CROMER et al (1981) mencionan que la relación Nitrógeno/Fósforo $(\mathrm{N} / \mathrm{P})$ para $\boldsymbol{E}$. grandis debe ser algo menor a 15 . SCHÖNAU y HERBERT (1982-83) establecen valores de 13. LAMB (1977) entrega valores de 10,4 para plantas de E. deglupta. LACELY et al (1966) dan valores de 11,9 para $\boldsymbol{E}$. grandis y los niveles de $\% \mathrm{~N}$ foliar lo fijan próximo al $2 \%$ o algo superior. BARROS y PRITCHETT (1979) consideran buenos u óptimos niveles de $2,2 \%$ de Nitrógeno. Todos estos autores no hacen referencia a niveles de Fósforo.

Por otra parte GONZALEZ, PENALVA y GOMEZ (1985) fijan valores críticos de Nitrógeno de $1,8 \%$ y suficiente cifras de $2 \%$. Para Fósforo, el valor crítico lo establecen en $0,12 \%$ y el nivel suficiente en $0,14 \%$. Por su parte GONZALEZ y GARCIA (1964) establecen los siguientes valores para Nitrógeno: deficiencia aguda $0,84 \%-1,05 \%$, deficiencia leve $1,05 \%$ $1,65 \%$, sin deficiencia $1,65 \% \cdot 1,80 \%$.

Aunque el nivel de nutrientes dado por el análisis foliar ha sido y es uno de los métodos más comunes para diagnosticar deficiencias nutricionales, existen otros como lo demuestra LAMB (1977). Este autor utiliza regresiones múltiples para explicar el crecimiento en altura en función de los contenidos foliares de nutrientes (Método 3). De esta manera establece que el $72 \%$ de crecimiento en altura está explicado por la concentración de los niveles de $\mathrm{N}$ y $\mathrm{P}$ y propone un nivel de 2,1\% de Nitrógeno como valor mínimo.

A partir de estos antecedentes, obtenidos en el extranjero y a los resultados de los distintos métodos de análisis empleados, se pueden establecer las siguientes conclusiones preliminares para los ensayos de El Almendro y Colcura. 


\section{CONCLUSIONES}

\section{El Almendro}

- $\quad$ El nivel de Nitrógeno alcanzado por los tratamientos 2, $\left(N_{1}\right) ; 8,\left(N_{2} P_{1}\right) ; 9,\left(N_{2} P_{1} B_{1} K_{1}\right)$; y $10,\left(N_{2} B_{1}\right)$; es bueno a óptimo. En el resto de los tratamientos existiria alguna deficiencia, sobre todo si se considera el valor propuesto por BARROS y PRITCHETT op. cit.

El nivel de Fósforo para todos los tratamientos está por debajo del valor crítico y distante del valor óptimo propuesto por GONZALEZ et al op. cit.

Para todos los tratamientos la relación N/P es superior a los valores señalados en la bibliografia, $\mathrm{Si}$ se considera el tratamiento $5,\left(\mathrm{~N}_{1} \mathrm{P}_{1} \mathrm{~B}_{1} \mathrm{~K}_{1}\right)$; como el mejor, se tiene una relación N/P igual a 21,9 . Es decir, un nivel de Nitrógeno próximo al $2 \%$ (nivel bueno) con un porcentaje de Fósforo de $0,09 \%$ (nivel deficiente). Para llegar a establecer un índice N/P igual a 13, el contenido foliar de Fósforo debería ser próximo a 0,15 (nivel óptimo).

- Los niveles óptimos encontrados a través del Método del Optimo Provisional Experimental, sólo constituye una primera aproximación a la solución de la problemática en estudio. A través de esta metodologia se establecen valores óptimos de Nitrógeno próximos al $2 \%$ y de Fósforo próximos al $0,087 \%$.

Para las condiciones de este ensayo y de acuerdo al análisis de regresión paso a paso (Stepwise), el nivel de Nitrógeno óptimo es 2,18\% y el de Fósforo alrededor del 0,10\%, lo que da una relación $\mathrm{N} / \mathrm{P}=21,8$.

\section{Colcura}

La mayoría de los tratamientos presentan un nivel de Nitrógeno bajo. Solamente los tratamientos $9,\left(\mathrm{~N}_{2} \mathrm{~B}_{2}\right)$; y $12,\left(\mathrm{P}_{1} \mathrm{~B}_{2}\right)$ sobrepasan el $2 \%$ de Nitrógeno.

- El nivel de Fósforo para todos los tratamientos está por debajo del valor crítico y distante

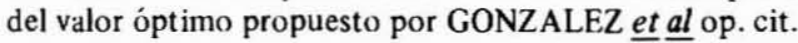

Para todos los tratamientos la relación $\mathrm{N} / \mathrm{P}$ es superior a los valores señalados por los distintos autores. Si se considera el tratamiento $4,\left(\mathrm{~N}_{1}\right)$; como el mejor, se tiene una relación N/P de 18,8. Es decir, este tratamiento presenta un porcentaje bajo de Nitrógeno y Fósforo.

A través del Optimo Provisional Experimental se pudieron establecer cifras referenciales correspondientes a $2,17 \%$ de Nitrógeno y $0,10 \%$ de Fósforo consideradas como buenas para el desarrollo de las plantas.

De acuerdo al Método 3, sólo el Nitrógeno juega un papel importante en el desarrollo de las plantas. El Fósforo por su bajo contenido foliar no seria significativo, en este caso. 


\section{REFERENCIAS BIBLIOGRAFICAS}

1. BARROS, N.F. y PRITCHETT, W.L. 1979. Effects of sources rales of Nitrogen on growth and composition of Eucalyptus grandis. Soil Crop. Sos. Soc. Fla Proc. (38). 101-104.

2. BEVIGE, D.I. y RICHARDS, B.N. 1971. Principles and practice of foliar analysis as a basis for crop logging in pine plantations II. Determination of critical phosphorus levels. Plant and soils (37): 159 . 169.

3. CABAÑ AS, C. 1982. Análisis comparativo entre fertilización mineral tradicional y por módulos en $E$. globulus. Tesis de Grado, Fac. de Cs. Agrarias, Veterinarias y Forestales. U. de Chile. 108 p.

4. CROMER, R.N. et al. 1981. Response of Eucalyptus species to fertilizer applied soon after planting at several sites. Aust. For. (44): 3 - 13.

5. FINKL, C. y SIMONSON, R.W. 1979. Phosphorus Cycle. In: "The Encycloped ia of Soil Science", Part I. Fairbridge R.W., Finkl C.W. Dowden. Hutchinson and Ross. Inc. p 370 - 376.

6. GONZALEZ, C. et al. 1973. La nutrición mineral de los vegetales. El análisis foliar y de savia. Metodologías y Objetivos. Fac. de Agronomía, Depto. de Frutales, Viñas, Universidad Católica de Chile.

7. GONZALEZ, E. PENALVA, F. y GOMEZ, C. 1985. Concentración foliar de nutrientes en E. globulus según el tratamiento fertilizante y época de su aplicación. Madrid. Anales del Instituto Nacional de Investigaciones Agropecuarias (INIA). Serie Forestal 1985. 47-56.

8. GONZALEZ, E. y GARCIA, J.J. 1964. Algunos aspectos de la nutrición nitrogenada en plantitas de Eucalyptus. Madrid. Anales del Instituto Forestal de Investigación de España. 81 - 101.

9. LACELY, C.J. et al. 1966. Growth and nutrient uptake by flooded gum seedlings subjected to variou: phosphate suppliers. Aus. For. (30:212 - 222).

10. LAMB, D. 1977. Relationship between growth and foliar nutrient concentration in Eucalyptus deglupte Plant and Soil (47): $495-508$.

11. PRADO, J.A. y WRANN, J. 1988. La importancia de la preparación del sitio y la fertilización en el esta blecimiento de plantaciones de Eucalyptus. En: Simposio Manejo Silvícola del Género Eucalyptus Junio 1988, Viña del Mar, Chile. Capítulo XII, Actas.

12. SCHÖNAU, A.P.G. 1981. The effects of fertilizing on the foliar nutrients concentration in Eucalyptu. grandis Fertilizer Res. (2): 73 - 87.

13. SCHÖNAU, A.P.G. y HERBERT, M.A. 1982. Relationship between growth rates and foliar concentra tions of Nitrogen, Phosphorus and Pootasium for $\boldsymbol{E}$. grandis. IUFRO XVII World Congress Kyoti Japan (1981) S. Afr. For. J. (120): $19-23$.

14. SCHÔNAU, A.P.G. y HERBERT, M.A. 1983. Relationship between growth rate, fertilizing and folia nutrient concentrations for $\boldsymbol{E}$. grandis. Fertilizer Res. (4): $369-380$.

15. SCHÖNAU, A.P.G. y HERBERT, M.A. 1988. Fertilizing Eucalypts at plantation establishment. En Simposio Manejo Silvícola del Género Eucalyptus, Junio 1988, Viña del Mar, Chile. Cap. X, Acta

16. UNIVERSIDAD DE CHILE - INSTITUTO FORESTAL. 1979. Areas cubiertas por ensayos de introdur ción de especies y ubicación de nuevas experiencias. Informe II. Proyecto CONAF / PNUD / FAO CHI-76-003 Investigación y Desarrollo Forestal. Actividad I 2.2 Introducción de Especies Forest: les. 81 p. Mapas. 